Revista Destaques Acadêmicos, Lajeado, v. 10, n. 3, 2018. ISSN 2176-3070

DOI: http://dx.doi.org/10.22410/issn.2176-3070.v10i3a2018.1782

http://www.univates.br/revistas

\title{
ASPECTOS EPIDEMIOLÓGICOS DE PACIENTES COM DIAGNOSTICO DE HANSENÍASE NA REGIÃO NORDESTE
}

\author{
Gleide Maria Gatto Bragança ${ }^{1}$, Nayane Nazareth Ferreira Lima ${ }^{2}$, \\ Luiz Cezar Lima Junior ${ }^{3}$, José Lucas Farias Wanderley ${ }^{4}$
}

\begin{abstract}
Resumo: Objetivo dessa pesquisa foi delinear/analisar os aspectos epidemiológicos da hanseníase nos estados da região nordeste entre o período de 2011 a 2015, avaliando em especialmente o número de casos, o coeficiente de prevalência, distribuição de acordo com o sexo e a faixa etária entre os estados da região nordeste. Trata-se de uma pesquisa com abordagem transversal retrospectiva, quantitativa do tipo descritiva, envolvendo pacientes com hanseníase ao diagnóstico. Os dados foram obtidos por meio de consulta no Sistema de Informação de Agravos de Notificação, fornecido pelo Departamento de Informática do Sistema Único de Saúde e estruturados no programa EXCEL versão 2010. Os resultados encontrados indicaram que dos 68.357 casos novos registrados, verificou-se redução no coeficiente de detecção geral que acompanhando o número de casos novos, do total, a maior parte era do sexo masculino. A faixa etária com maior índice foi de 35 a 49 anos de idade. Conclui-se em relação aos números de casos diagnosticados percebemos uma redução desses casos novos no estado de Sergipe, sendo que o coeficiente de prevalência desse estado permaneceu no parâmetro de baixo preconizado pelo MS, seguido de mais três estados: Rio Grande do Norte, Alagoas e Paraíba. Pode-se observar a prevalência do sexo masculino, assim como um maior índice de faixa etária de 35 a 49 anos tendo uma maior predominância no estado do Maranhão.
\end{abstract}

Palavras-chave: Hanseníase. Epidemiologia. Saúde pública.

1 Professora de Dermatologia do curso de Medicina da Universidade Tiradentes (UNIT), graduação em Medicina pela Universidade Federal de Sergipe (UFS), Mestre em Saúde e Ambiente pela Universidade Tiradentes, E-mail: gleidegattodemato@hotmail.com.

2 Graduada em medicina pela Universidade Tiradentes (UNIT), E-mail: nayanenf_lima@hotmail.com

3 Mestre em Desenvolvimento e Meio Ambiente, pela Universidade Federal de Sergipe (UFS) Especialista em Bases Nutricionais da Atividade Física, bacharel em Educação Física pela Universidade Tiradentes (UNIT) e graduado em Licenciatura pela Claretiano. E-mail: llima1305@hotmail.com.

4 Graduado em Medicina pela Universidade Tiradentes (UNIT), E-mail: lucasfarias775@hotmail.com 


\section{INTRODUÇÃO}

A Hanseníase é uma infecção bacteriana, de caráter contagioso e evolução crônica. Causada pelo Mycobacterium leprae (M. leprae) ou bacilo de Hansen, parasita intracelular obrigatório, que apresenta uma grande preferência por células cutâneas e nervosas. Este bacilo apresenta alta infectividade e virulência com uma baixa patogenicidade, ou seja, a doença tem capacidade de infectar um grande número de indivíduos, mas poucos adoecem (AZULAY; AZULAYABULAFIA, 2015; LASTÓRIA et al., 2014; LYON; GROSSI, 2013; PORTO et al., 2016; SOUZA, 2017).

Lyon e Grossi (2013) afirmam que o diagnóstico da hanseníase é essencialmente clínico, somado à análise epidemiológica, baseado na história, nas condições de vida do indivíduo e no exame das lesões cutâneas e/ou de algum comprometimento nervoso. Em relação às manifestações clínicas, relaciona-se com a imunidade do hospedeiro ao bacilo, em comparação à sua capacidade de penetração e multiplicação (AZULAY; AZULAY-ABULAFIA, 2015; LASTÓRIA et al., 2014).

De acordo Ministério da Saúde, 2016, as lesões cutâneas se caracterizam por manchas hipocrômicas ou eritematosas, pápulas, infiltrações, tubérculos e nódulos; somados à diminuição ou à ausência de sensibilidade. As lesões neurológicas apresentam-se por acometimento das terminações nervosas sensitivas, motoras e autonômicas e/ou por troncos nervosos periféricos, causando dor e/ou espessamento (neurite), diminuição e/ou perda de sensibilidade e de força (AZULAY; AZULAY-ABULAFIA, 2015; LASTÓRIA et al., 2014; LYON; GROSSI, 2013).

A doença é considerada endêmica nos países tropicais e subtropicais, resultando em torno de 106 países endêmicos. De acordo com Organização Mundial de Saúde (OMS, 2016) há uma redução gradual na detecção de casos novos nos últimos 10 anos: de 265.661 em 2006 para 210.758 em 2015. O continente americano seguiu essa tendência com uma queda de $47.612 \mathrm{em} 2006$ para 28.806 casos em 2015. Contudo, o Brasil está em segundo lugar no número de casos novos, perdendo somente para a Índia com os seguintes valores 31.044 (2013); 31.064 (2014) e 26.395 (2015) (AZULAY; AZULAY-ABULAFIA, 2015; LASTÓRIA et al., 2014; WHO, 2016).

Apesar de ter uma evolução crônica, ocorrem períodos de agudização durante qualquer fase da doença ou do tratamento, chamadas de reações hansênicas. Elas mantêm uma relação com a imunidade do indivíduo, assim variam de intensidade e de órgão atingido, sendo a principal causa de morbidade e de incapacidade do paciente. Classificam-se em dois tipos: reação tipo 1 (reação reversa) e reação tipo 2 (eritema nodoso hansêmico). A primeira, mediada por células (Th1), caracteriza-se por hiperestesias das lesões antigas que se tornam eritematosas, intumescidas e infiltradas, por surgimento de novas lesões com as mesmas características e neurites frequentes que 
podem ser silenciosas. Enquanto na segunda, mediada por imunocomplexos (Th2), as lesões cutâneas antigas permanecem inalteradas e surgem nódulos inflamatórios simétricos, em qualquer região, ou lesões em alvo do tipo eritema multiforme acompanhados de comprometimento sistêmico (AZULAY; AZULAY-ABULAFIA, 2015; LASTÓRIA et al., 2014; MINISTÉRIO DA SAÚDE, 2016; SOUZA, 2017).

Vários foram os tipos de classificação, utilizando desde os aspectos clínicos, bacteriológico, imunológico e histopatológico (AZULAY; AZULAYABULAFIA, 2015; LASTÓRIA et al., 2014; LYON; GROSSI, 2013). O Ministério da Saúde e a Organização Mundial da Saúde utilizam uma classificação para fins terapêuticos, de acordo com o número de lesões: os paucibacilares (PB) - até cinco lesões; os multibacilares (MB) - mais de cinco lesões. Foi definido o tratamento da hanseníase com a poliquimioterapia (PQT) - três drogas: dapsona, rifampicina e clofazimina - com diferentes combinações e tempo do tratamento de acordo com o tipo a ser tratado (AZULAY; AZULAYABULAFIA, 2015; LASTÓRIA et al., 2014; LYON; GROSSI, 2013).

Desde a criação e disponibilização da PQT, a hanseníase começou a apresentar declínios na sua epidemiologia. Somado a isso, várias foram as metas utilizadas para o controle da hanseníase desde metas de eliminação (2000-2005) a metas de redução da carga da doença (2006-2010 e 2011-2015). A eliminação da hanseníase como problema de saúde pública foi alcançada em 2000 em escala mundial e até 2005 na maioria dos países. No entanto, o Brasil não alcançou as metas e a hanseníase ainda se mantém um problema de saúde pública (SOUZA, 2017; WHO, 2016a, 2016b).

Conforme foi exposto, a hanseníase ainda é, no Brasil, considerada um problema de saúde pública e uma doença endêmica devido a fatores como: diagnósticos tardios por parte dos profissionais de saúde (o que indica um despreparo e precariedade do serviço) e ao pouco esclarecimento da população quanto à identificação, à prevenção e ao tratamento da doença (provocando assim o abandono precoce do tratamento e a estereotipagem do indivíduo doente pelas incapacidades físicas).

Com uma distribuição heterogênea, a hanseníase no Brasil reproduz as desigualdades socioeconômicas entre as diferentes regiões do país (BARBOSA; ALMEIDA; SANTOS, 2014; SOUZA, 2017). Nos últimos anos, foram observadas as maiores concentrações em regiões como o Norte, o Nordeste e o Centro-Oeste (BARBOSA; ALMEIDA; SANTOS, 2014; SOUZA, 2017). De acordo com Souza (2017), estudos mostraram 10 clusters no Brasil, áreas com grande aglomeração de casos e índices de transmissão, com mais de 50\% dos casos. No entanto, ocorreu uma redução do munícipios endêmicos de 1.173 para 621 em 2013 (AZULAY; AZULAY-ABULAFIA, 2015; LASTÓRIA et al., 2014). Na região Nordeste se concentra um grande número de casos novos (primeiro lugar desde 2001), sendo utilizado para monitoramento da endemia o número de casos de hanseníase em menores de 15 anos (AZULAY; AZULAY- 
ABULAFIA, 2015; LASTÓRIA et al., 2014). Devido à importância dessa doença, esta pesquisa delineou-se a partir dessa problemática, tendo como objetivo analisar os aspectos epidemiológicos da hanseníase nos estados da região nordeste entre o período de 2011 a 2015, avaliando em especialmente o número de casos, o coeficiente de prevalência, distribuição de acordo com o sexo e a faixa etária entre os estados da região nordeste. Pois trata-se de um estudo relevante dentro do contexto epidemiológico, da saúde envolvendo pacientes diagnosticados com hanseníase.

\section{MÉTODOS}

Trata-se de um estudo epidemiológico, de natureza descritiva, do tipo transversal, com abordagem quantitativa envolvendo pacientes diagnosticados com hanseníase.

A pesquisa é epidemiológica por descrever a frequência e a distribuição de eventos ligados à saúde em populações específicas. É descritiva e quantitativa por corresponder à distribuição de frequência das doenças e dos agravos à saúde em função de variáveis ligadas ao tempo, ao espaço e à pessoa, possibilitando o detalhamento do perfil epidemiológico, com vistas à promoção da saúde. É retrospectiva por se tratar de pesquisa cuja fonte de coleta de dados está restrita a documentos escritos, constituindo o que se denomina de fontes secundárias.

A população selecionada foi composta por pacientes com diagnóstico confirmado de hanseníase e notificados ao Sistema de Informações de Agravos de Notificação (SINAN). O período estudado foi de 2011 a 2015. Este intervalo de tempo foi delimitado por abranger o período entre o ponto inicial das novas diretrizes definidas pelo Plano Integrado (2011-2015) de ações de eliminação e o controle da hanseníase. A técnica de coleta de dados utilizada foi consulta documental na base de dados do DATASUS, que contém sistemas de informações em saúde disponíveis pela Internet, no website http:/ / www. datasus.gov.br que foi acessado entre o período de 15/03/2017 a 05/04/2017. Os dados foram organizados em planilhas do software Microsoft Excel 2010 e avaliadas através de estatística descritiva.

A caracterização epidemiológica foi realizada com base nos indicadores de monitoramento e avaliação da hanseníase para todos os anos do estudo. Os indicadores selecionados foram àqueles preconizados pelo Ministério da Saúde, segundo Portaria $n^{\circ} 3.125$ de 2010, para avaliação e monitoramento da hanseníase: coeficiente de prevalência por 10.000 hab.

\section{RESULTADOS E DISCUSSÕES}

A hanseníase é uma doença infectocontagiosa causada pelo Mycobacterium leprae ou bacilo de Hansen, possuindo uma alta infectividade e virulência e baixa patogenicidade, sendo considerada um importante problema 
de saúde pública. Em 2011, 228.474 casos foram detectados no mundo. O Brasil ocupa o segundo lugar em número absoluto de casos, atrás apenas da Índia, sendo único país que não atingiu a meta de eliminação da doença como problema de saúde pública, definida pela prevalência menor que 1 caso/10.000 habitantes. Com uma distribuição heterogênea, a doença reproduz as desigualdades socioeconômicas entre as diferentes regiões do país.

Sobre a região Nordeste, sempre ocupou os primeiros lugares em número de casos novos. De acordo com a tabela 1, foram notificados 68.357 casos novos na região Nordeste, sendo uma média de 13.671 casos/ano. Com $14.333 \mathrm{em}$ 2011 e 12.760 em 2015, mostrando uma redução (de aproximadamente 11\%), que acompanhou o nível nacional, mostrado na tabela abaixo.

Tabela 1: Distribuição de casos novos de Hanseníase por Região, nos períodos 2011-2015

\begin{tabular}{c|c|c|c|c|c}
\hline Ano/Região & Norte & Nordeste & $\begin{array}{c}\text { Centro- } \\
\text { Oeste }\end{array}$ & Sul & Sudeste \\
\hline 2011 & 6.873 & 14.333 & 6.126 & 1.374 & 5.837 \\
2012 & 6.876 & 14.263 & 5.432 & 1.349 & 5.813 \\
2013 & 6.052 & 13.389 & 4.811 & 1.171 & 5.821 \\
2014 & 6.052 & 13.612 & 4.520 & 1.025 & 6.213 \\
2015 & 5.147 & 12.760 & 4.013 & 1.013 & 5.623 \\
\hline
\end{tabular}

Fonte: dados obtidos do Departamento de Informática do Sistema Único de Saúde (Datasus/MS), alimentado pelo SINAN - Sistema de Informações de Agravos de Notificação. MS/SVS/CGHDE - Coordenação Geral de Hanseníase e Doenças em Eliminação

A Tabela 2, a seguir, mostra que, no período de 2011 e 2012, o coeficiente de detecção maior que 20/100.000 hab., considerado muito alto pelos parâmetros do MS, apareceu em cinco estados (SE, CE, PE, PI e MA). Em 2013, o Estado de Sergipe apresentou redução consistente atingindo o quarto menor coeficiente da região em 2015 (16,05/100.000 hab). Nos anos seguintes, os outros quatro estados mantiveram valores acima de 20/100.000 hab, apesar da inclinação descendente sendo que o coeficiente mais alto refere-se ao Estado do Maranhão.

Ainda em análise da Tabela 2, os Estados que apresentaram um coeficiente de prevalência menor que 1 para cada 10.000 habitantes em 2015, foram Rio Grande do Norte $(0,49)$, Sergipe $(0,95)$, Alagoas $(0,65)$ e Paraíba $(0,85)$, considerados baixos de acordo com o ministério da saúde. 
Tabela 2: Distribuição de números de casos novos e do coeficiente de prevalência de Hanseníase nos Estados do Nordeste no período 2011-2015

\begin{tabular}{c|c|c|c|c|c|c|c|c|c}
\hline $\begin{array}{c}\text { Ano/ } \\
\text { Estados NE }\end{array}$ & RN & AL & SE & PB & PI & CE & PE & BA & MA \\
\hline 2011 & & & & & & & & & \\
Casos novos & 265 & 405 & 422 & 703 & 1139 & 2000 & 2724 & 2729 & 3946 \\
Coef. Prev. & 0,04 & 0,05 & 0,02 & 0,07 & 0,12 & 0,09 & 0,1 & 0,16 & 0,16 \\
População & 3.302 .061 & 3.256 .277 & 2.145 .945 & 3.852 .082 & 3.158 .753 & 8.641 .877 & 9.062 .506 & 14.865 .405 & 6.670 .977 \\
2012 & & & & & & & & & \\
Casos novos & 316 & 465 & 468 & 708 & 1099 & 2139 & 2575 & 2594 & 3899 \\
Coef. Prev. & 0,09 & 0,05 & 0,08 & 0,15 & 0,2 & 0,07 & 0,13 & 0,15 & 0,19 \\
População & 3.338 .489 & 3.279 .289 & 2.171 .137 & 3.883 .822 & 3.172 .210 & 8.711 .659 & 9.136 .697 & 14.957 .177 & 6.734 .353 \\
2013 & & & & & & & & & \\
Casos novos & 275 & 347 & 379 & 632 & 1037 & 2070 & 2588 & 2234 & 3827 \\
Coef. Prev. & 0,08 & 0,06 & 0,04 & 0,15 & 0,2 & 0,05 & 0,19 & 0,13 & 0,17 \\
População & 3.373 .989 & 3.300 .935 & 2.195 .662 & 3.914 .421 & 3.184 .166 & 8.778 .576 & 9.208 .550 & 15.044 .137 & 6.794 .301 \\
2014 & & & & & & & & & \\
Casos novos & 274 & 343 & 403 & 575 & 1133 & 1974 & 2556 & 2650 & 3704 \\
Coef. Prev. & 0,29 & 0,16 & 0,21 & 0,42 & 0,48 & 0,25 & 0,61 & 0,32 & 0,86 \\
População & 3.408 .575 & 3.321 .291 & 2.219 .563 & 3.943 .847 & 3.193 .962 & 8.843 .556 & 9.278 .160 & 15.126 .447 & 6.850 .833 \\
2015 & & & & & & & & & \\
Casos novos & 267 & 351 & 360 & 523 & 1012 & 1790 & 2383 & 2540 & 3534 \\
Coef. Prev. & 0,49 & 0,65 & 0,95 & 0,85 & 2,09 & 1,46 & 1,87 & 1,12 & 3,78 \\
População & 3.442 .158 & 3.340 .528 & 2.242 .948 & 3.972 .175 & 3.203 .248 & 8.905 .190 & 9.345 .638 & 15.203 .851 & 6.904 .298 \\
\hline
\end{tabular}

Fonte: dados obtidos do Departamento de Informática do Sistema Único de Saúde (Datasus/ MS),alimentado pelo SINAN - Sistema de Informações de Agravos de Notificação. MS/ SVS/CGHDE - Coordenação Geral de Hanseníase e Doenças em Eliminação. Coeficiente de prevalência por 10.000 hab. Coeficiente de detecção de casos novos por 100.000 hab Legenda: Coef.: Coeficiente. (-) Dado numérico igual a 0 não resultante de arredondamento; $(0 ; 0,0)$ Dado numérico igual a 0 resultante de arredondamento de um dado originalmente positivo

No que diz respeito à análise do sexo (tabela 3), nos 5 anos estudados, de modo geral ocorreu uma maior incidência do sexo masculino (MA, PI, CE, PB e BA). Apesar de se observar que Estados como Rio Grande do Norte, Pernambuco, Sergipe apresentaram razão próximo a 1, indicando não predileção por um sexo, sendo os dois igualmente acometidos, enquanto, observou-se no Estado do Alagoas uma predominância feminina $(51,8 \%)$. 
Tabela 3: Distribuição de casos novos por sexo entre os Estados do Nordeste, nos anos de 2011-2015

\begin{tabular}{l|l|l|l}
\hline Estados & Masculino & Feminino & RM \\
\hline MA & $10632(56,2 \%)$ & $8278(43,8 \%)$ & 1,28 \\
PI & $2917(53,8 \%)$ & $2503(46,1 \%)$ & 1,16 \\
CE & $5627(56,4 \%)$ & $4344(43,7 \%)$ & 1,29 \\
RN & $694(49,7 \%)$ & $703(50,3 \%)$ & 0,98 \\
PB & $1684(53,6 \%)$ & $1457(46,4 \%)$ & 1,15 \\
PE & $6359(49,6 \%)$ & $6467(50,4 \%)$ & 0,98 \\
AL & $921(48,2 \%)$ & $989(51,8 \%)$ & 0,93 \\
SE & $1015(49,9 \%)$ & $1017(50 \%)$ & 0,99 \\
BA & $6595(51,7 \%)$ & $6150(48,2 \%)$ & 1,07 \\
\hline
\end{tabular}

Fonte: dados obtidos do Departamento de Informática do Sistema Único de Saúde (Datasus/MS), alimentado pelo SINAN - Sistema de Informações de Agravos de Notificação. MS/SVS/CGHDE - Coordenação Geral de Hanseníase e Doenças em Eliminação. Legenda: n(\%) - número absoluto (porcentagem calculada sobre o valor total).

Ao ser analisado o critério faixa etária, conforme Tabela 4, os registros mostraram um maior índice na faixa etária de 35 a 49 anos de idade em praticamente todos os Estados, com exceção de Piauí, Ceará e Rio Grande do Norte que se observou uma maior proporção entre a faixa etária 50 a 64 anos. A menor ocorrência ocorreu em pacientes $\geq 80$ anos de idade.

Tabela 4: Distribuição de casos novos por faixa etária entre os Estados do Nordeste, nos anos de 2011-2015

\begin{tabular}{|c|c|c|c|c|c|c|c|c|c|}
\hline \multirow{2}{*}{$\begin{array}{l}\text { Faixa } \\
\text { Etária }\end{array}$} & \multicolumn{9}{|c|}{ Estados n(\%) } \\
\hline & MA & PI & CE & RN & PB & PE & $\mathrm{AL}$ & $\mathrm{SE}$ & BA \\
\hline$<15$ anos & $1878(9,9)$ & $373(6,9)$ & $583(5,8)$ & $98(7)$ & $180(5,7)$ & $1358(10,6)$ & $126(7,6)$ & $133(6,5)$ & $971(7,6)$ \\
\hline 15-19 anos & $1142(6,0)$ & $301(5,6)$ & $391(3,9)$ & $37(2,6)$ & $126(4)$ & $669(5,2)$ & $106(5,5)$ & $93(4,6)$ & $614(4,8)$ \\
\hline 20-34 anos & $4520(23,9)$ & $1066(19,7)$ & $1616(16,2)$ & $214(15,3)$ & $687(21,9)$ & $2674(20,9)$ & $446(23,3)$ & $423(20,8)$ & $2741(21,5)$ \\
\hline 35-49 anos & $4499(23,8)$ & $1373(25,3)$ & $2698(27,1)$ & $376(26,9)$ & $817(26)$ & $3403(26,6)$ & $512(26,8)$ & $548(27)$ & $3452(27,1)$ \\
\hline 50-64 anos & $4104(21,7)$ & $1391(25,7)$ & $2720(27,3)$ & $413(29,6)$ & $774(24,6)$ & $3001(23,4)$ & $486(25,4)$ & $501(24,1)$ & $3134(24,6)$ \\
\hline 65-79 anos & $2308(12,2)$ & $740(13,7)$ & 1599(16) & $215(15,4)$ & $457(14,5)$ & $1446(11,6)$ & $202(10,6)$ & $271(13,3)$ & $1490(11,7)$ \\
\hline$\geq 80$ anos & $458(2,4)$ & $176(3,2)$ & $365(3,7)$ & $44(3,1)$ & $100(3,2)$ & $257(2,0)$ & $33(1,7)$ & $63(3,1)$ & $345(2,7)$ \\
\hline
\end{tabular}

Fonte: dados obtidos do Departamento de Informática do Sistema Único de Saúde (Datasus/MS), alimentado pelo SINAN - Sistema de Informações de Agravos de Notificação. MS/SVS/CGHDE Coordenação Geral de Hanseníase e Doenças em Eliminação. Legenda: n(\%) - número absoluto (porcentagem calculada sobre o valor total). 
A análise dos dados mostrou uma redução do número de casos em Sergipe, seguida pelos demais estados do Nordeste no período de 2011 a 2015. A presente pesquisa verificou, também, que essa redução acontece a nível nacional e regional. Em concordância, de acordo com a OMS (2016) os números absolutos de novos casos detectados nos últimos 10 anos (2006-2015) apresentam uma queda gradual global.

Ao longo do período estudado, o coeficiente de prevalência esteve abaixo do proposto pela Organização Mundial da Saúde, desde 1991, a Assembleia Mundial de Saúde decidiu "eliminar a Hanseníase como um problema de saúde pública até o ano 2000, com a redução da prevalência global da doença para menos de 1 caso por 10.000 hab (WHO, 2016a, 2016b). Ao se analisar isoladamente cada ano de estudo, observou-se, que no ano de 2015, ocorreu um aumento do coeficiente em alguns estados, chegando a um índice médio (1,0 a 4,9/10.000 hab.) no estado do Maranhão. Os resultados sugeriram uma possível subnotificação, seguido de um aumento no rastreio, já que a partir de 2011, medidas de controle foram expandidas e fortalecidas com a criação da Coordenação Geral de Hanseníase e Doenças em Eliminação e, em 2012, do Plano Integrado de Ações Estratégicas de Eliminação da Hanseníase e outras doenças incluindo esquistossomose, filariose linfática, geohelmintíases, oncocercose e tracoma. (MINISTÉRIO DA SAÚDE, 2011)

Apesar disso, como o coeficiente de prevalência leva em consideração apenas o número de casos registrados em tratamento no dia 31 de dezembro do ano avaliado, não é o melhor indicador para avaliar a redução da doença, já que pacientes que receberam alta após seis a nove meses de tratamento não são incluídos no cálculo (RIBEIRO et al., 2014).

Durante o período analisado, o sexo masculino prevaleceu sobre o feminino com pequenas diferenças em alguns estados, corroborando dados nacionais e internacionais sobre o tema. Esse é um resultado mostrado por vários estudos, não só para a hanseníase como para outras doenças infecciosas ou não. Isso se deve a um maior relacionamento com a sociedade, a uma menor preocupação com aspectos relacionados ao seu corpo e sua saúde e a dificuldade de acesso aos serviços de públicos de saúde; essas últimas justificativas explicariam o porquê do leve aumento no sexo feminino, considerado indicador de acesso aos serviços de saúde (SILVA; TOLEDO; GELATTI, 2016; BARBOSA; ALMEIDA; SANTOS, 2014; OLIVEIRA; LEÃO; BRITTO, 2014; SOUSA et al., 2012).

Quando se considera a faixa etária como variável de análise, a maior prevalência deu-se 35 a 49 anos, concentrando a maior parte da população economicamente ativa, como afirma Barbosa, Almeida e Santos, (2014). Esse fato revela um risco de exclusão da cadeia produtiva, já que esse público enfrenta dificuldade em trabalhar, manter-se no trabalho ou de enfrentar um novo trabalho, pois mesmo hoje a hanseníase é vista de forma negativa, 
ainda como estigma, além de provocar um alto custo social (SILVA; TOLEDO; GELATTI, 2016; SANTOS FILHO, 2012; SOUSA et al., 2012).

\section{CONCLUSÕES}

A partir dos estudos obtidos observa-se alguns pontos relevantes, a utilização de dados secundários mostrou-se ser uma delas, muitas vezes pelas inconsistências causadas pela subnotificação. Além de que o estudo não desenvolveu o conhecimento das características geoespaciais o que poderia justificar as diferenças observadas nos Estados. Com isso, vale salientar a importância da sistematização de um banco de dados na vigilância epidemiológica, armazenando informações que contribuam para a diminuição e controle da hanseníase.

Em relação aos números de casos diagnosticados percebe-se uma redução desses casos novos no estado de Sergipe, sendo que o coeficiente de prevalência desse estado permaneceu no parâmetro de baixo preconizado pelo MS, seguido de mais três estados: Rio Grande do Norte, Alagoas e Paraíba.

Pode-se observar a prevalência do sexo masculino, assim como um maior índice de faixa etária de 35 a 49 anos tendo uma maior predominância no estado do Maranhão.

\section{REFERÊNCIAS}

AZULAY, R. D.; AZULAY-ABULAFIA, L. Doenças Causadas por Agentes Biológicos - Micobacterioses. Dermatologia. 6 rev. e atual ed. Rio de Janeiro: Guanabara Koogan, 2015.

BARBOSA, D. R. M.; ALMEIDA, M. G.; SANTOS, A. G. Dos. Características epidemiológicas e espaciais da hanseníase no Estado do Maranhão, Brasil, 2001-2012. Medicina (Ribeirão Preto. Online), v. 47, n. 4, p. 347-356. 30 dez. 2014.

LASTÓRIA, J. C. et al. Leprosy: review of the epidemiological, clinical, and etiopathogenic aspects - Part 1. Anais Brasileiros de Dermatologia, v. 89, n. 2, p. 205-218, abr. 2014.

LYON, S.; GROSSI, M. A. Hanseníase. Rio de Janeiro: Medbook, 2013.

MINISTÉRIO DA SAÚDE. Plano nacional de eliminação da Hanseníase em nível municipal: 2006-2010. [S.l.], 2006. Disponível em: <http://bases.bireme.br/cgi-bin/wxislind.exe/iah/online/?IsisScript=iah/iah.xis\&src=google\&base=LILACS\&lang=p\&nextAction=lnk\&exprSearch=468744\&indexSearch=ID>. Acesso em: 29 abr. 2017.

Plano integrado de ações estratégicas de eliminação da hanseníase, filariose, esquistossomose e oncocercose como problema de saúde pública, tracoma como causa de cegueira e controle das geohelmintíases : plano de ação 2011-2015. $1^{\mathrm{a}}$ ed. Brasília, DF: [s.n.], 2011. 
OLIVEIRA, J. C. F. De; LEÃO, A. M. M.; BRITTO, F. V. S. Análise do perfil epidemiológico da hanseníase em Maricá, Rio de Janeiro: uma contribuição da enfermagem. Revista Enfermagem UERJ, v. 22, n. 6. 23 dez. 2014. Disponível em: <http:/ / www.e-publicacoes.uerj.br/index.php/enfermagemuerj/article/ view /13400>. Acesso em: 6 maio 2017.

PORTO, M. J. et al. ANALISE DOS NÚMEROS DE CASOS NOTIFICADOS DE HANSENÍASE PELO SINAN NA BAHIA. Revista Enfermagem Contemporânea, v. 4, n. 2, 8 mar. 2016. Disponível em: <https:/ / www5.bahiana.edu.br/index.php/ enfermagem/article/view/455>. Acesso em: 20 maio 2017.

SANTOS FILHO, R. C. Dos. Perfil clínico-epidemiológico da hanseníase no município de Irecê-Bahia, período 2001 a 2011. [S.l.]: [s.n.], 2012. Monografia (Conclusão de curso) - Universidade Federal da Bahia, Faculdade de Medicina, Salvador, 2012. Disponível em: <https:/ / www.repositorio.ufba.br/ri/handle/ ri/8105>. Acesso em: 29 abr. 2017.

SILVA, M. N. Da; TOLEDO, B. J.; GELATTI, L. G. PERFIL EPIDEMIOLÓGICO DE PACIENTES PORTADORES DE HANSENÍASE EM URUAÇU-GO. Fasem Ciências, v. 7, n. 1, p. 18-28, 24 fev. 2016.

SOUSA, M. W. G. De et al. Epidemiological Profile of Leprosy in the Brazilian state of Piauí between 2003 and 2008. Anais Brasileiros de Dermatologia, v. 87, n. 3, p. 389-395. jun. 2012.

SOUZA, E. A. De. Hanseníase, risco e vulnerabilidade: perspectiva espaço-temporal e operacional de controle no Estado da Bahia, Brasil. [S.l.]: [s.n.], 2017. Tese (Doutorado) - Universidade Federal do Ceará, Programa de Pós-Graduação em Saúde Pública, Fortaleza, 2017. Disponível em: <http:/ / www.repositorio.ufc.br/handle/ riufc/21646>. Acesso em: 20 maio 2017.

WHO | Operational Manual 2016 - Global Leprosy Strategy 2016-2020. WHO, [S.1.], 2016a. Disponível em: <http:/ / www.who.int/lep/resources/9789290225256/en/>. Acesso em: 23 abr. 2017. 